\title{
ANÁLISE DO PODER DE PREVISÃO DA TAXA DE DIVIDENDO SOBRE O RETORNO E DIVIDENDO NO IBOVESPA
}

\section{AN ANALYSIS OF THE PREDICTIVE POWER OF DIVIDEND RATE ON THE RETURN AND DIVIDEND IN THE BOVESPA INDEX}

\author{
José Guilherme Chaves Alberto \\ Universidade Fumec, MG \\ guilhermezaragoza@gmail.com \\ Aureliano Angel Bressan \\ UFMG \\ bressan@face.ufmg.br \\ Robert Aldo Iquiapaza Coaguila \\ UFMG \\ riquiapaza@gmail.com \\ Flávio Dias Rocha \\ Centro Universitário UNA, MG \\ flaviorocha@prof.una.br \\ Luiz Henrique Cota \\ Empresa PlanoTI, MG \\ luizhenriquecota@gmail.com
}

Submissão: 12/02/2013

Aprovação: 26/06/2013

\section{RESUMO}

A eficiência da taxa de dividendo em prever o movimento futuro das ações ou do dividendo sempre foi muito discutida, tanto no meio acadêmico como nos mercados financeiros. Analistas de investimentos defendem que a taxa de dividendo pode ser usada como estratégia para obtenção de retornos anormais; por outro lado, pesquisadores ainda têm uma visão bem contraditória a respeito do poder de previsão e da eficiência dessa estratégia. Desse modo, este trabalho busca analisar se a taxa de dividendo tem a capacidade de prever o retorno, a variação do dividendo futuro ou ambos. Foram analisados dados semestrais, em moeda original e corrigidos pela inflação, no período de 1994 a 2010, sendo a carteira do Ibovespa utilizada como proxy do mercado. Os resultados demonstraram que a taxa de dividendo é eficiente em prever a variação do dividendo por ação da carteira do Índice Bovespa. Em relação ao retorno, os resultados não foram significativos na previsão.

Palavras-chave: Taxa de dividendo; Retorno ativo; Crescimento dividendo; Previsão de longo prazo e curto prazo; Vetor auto-regressivo. 


\begin{abstract}
The efficiency of the dividend yield in predicting future changes in stock prices or dividends has always been discussed both in academic circles and financial markets. On the one hand, investment analysts argue that a dividend yield strategy can provide abnormal returns. On the other hand, researchers are still skeptical on this strategy's predictive power and efficiency. Thus, this paper aims to analyze whether dividend yield predicts stock returns, dividend changes, or both. Semiannual data, in original currency and corrected for inflation, was analyzed for the period from 1994 to 2010, and the Ibovespa was used as a market proxy. The results have shown that the dividend yield is efficient in predicting changes in dividends per share in the Bovespa Index stocks. Nevertheless, when tested for its predicting power for stock returns, the results were not statistically significant.
\end{abstract}

Keywords: Dividend rate; Active return; Dividend growth; Short-term and long-term forecasts; Vector autoregressive. 


\section{INTRODUÇÃO}

O desenvolvimento do mercado de capitais brasileiro com o processo de estabilização econômica, a partir da implementação do Plano Real em 1994, levou um número cada vez maior de investidores a buscar estratégias de investimento visando obter maior rentabilidade. Uma das abordagens mais divulgadas e amplamente aceitas é a avaliação relativa das empresas, popularmente conhecida como análise de múltiplos, sendo a taxa de dividendo (dividend yield) uma das mais utilizadas pelos investidores no mercado acionário.

A utilização da taxa de dividendo como ferramenta de seleção de ativos se fundamenta na expectativa de que as ações que possuem alta taxa de dividendo tendem a estar subvalorizadas, gerando retorno superior ao mercado, suposição essa contrária à Hipótese do Mercado Eficiente, que defende a impossibilidade de obter retornos acima do mercado, pois todas as informações já estão refletidas nos preços dos ativos de alguma maneira (FAMA, 1970).

Estudos empíricos anteriores apontam que, se a taxa de dividendo tende a se ajustar para valores médios históricos, ou seja, segue um processo estacionário, o ajuste pode ocorrer nos dividendos futuros pagos pelas empresas, nos preços dos ativos ou em ambos.

Trabalhos como os de Miller e Modigliani (1961); Graham, Dodd e Cottle (1962); Shefrin e Statman (1984); Campbell (2003); Cochrane (2008); Chen (2009); e Engsted e Pedersen (2010) analisaram diferentes abordagens relacionadas a dividendos como: a eficiência da estratégia de comprar ações com alta taxa de dividendo, a preferência dos acionistas em receber ou não dividendos e o poder de previsão da taxa de dividendo em relação ao retorno e a variação do dividendo futuro por ação. No Brasil destacam-se os estudos de Bueno (2002) e Neto e Saito (2002).

Dado esse contexto, este trabalho tem como objetivo, a partir do estudo da carteira teórica do índice Bovespa no período de 1994 a 2010, investigar se a taxa de dividendo possui um comportamento estacionário ao longo do tempo e analisar a capacidade preditiva em relação à variação futura do preço do ativo e do dividendo pago por ação, utilizando modelos de Vetores Auto-Regressivos - VAR. Os cálculos, da variação do retorno e do dividendo por ação, foram realizados em intervalos de tempo semestrais em taxa real e nominal. $\mathrm{O}$ valor do dividendo foi obtido pela soma dos dividendos e dos juros sobre capital próprio.

A escolha do período de 1994 a 2010 foi devido ao êxito do plano Real no controle da inflação, conforme ressaltado em diversos trabalhos como Holanda (2003); Grasel (2005); Fontes, Arbex e Silva (1998).

O tema é relevante, tendo em vista que o estudo aumenta a base de conhecimento sobre o mercado acionário brasileiro e pode ajudar na tomada de decisão dos investidores quanto à eficiência da utilização da taxa de dividendo em estratégias de investimentos em ativos.

O trabalho está estruturado em cinco seções, incluindo esta introdução. Na Seção 2, apresenta-se a revisão da literatura; na Seção 3, descreve-se a metodologia; na Seção 4, desenvolve-se a análise dos dados e a discussão dos resultados; e na Seção 5, expõem-se as conclusões do trabalho. 


\section{REFERENCIAL TEÓRICO}

\subsection{Taxa de Dividendo (dividend yield)}

A taxa de dividendo é um dos múltiplos mais utilizados na avaliação relativa das empresas. De acordo com Damodaran (2007) a avaliação relativa tem como principal objetivo precificar um ativo com base na forma como ativos comparáveis são avaliados pelo mercado, sendo que a correta utilização dessa técnica irá depender de como o mercado está pagando por ativos similares. Para uma correta utilização dos múltiplos é também necessária uma análise da sua distribuição média histórica.

A taxa de dividendo (dividend yield) corresponde ao percentual entre o dividendo pago por ação de uma empresa e o valor de mercado dessa empresa (MATIAS, 2009). Seu cálculo é realizado por meio da seguinte fórmula:

$D Y_{t}=D P A_{t} / P_{t}$

Sendo:

$\mathrm{DYt}=$ taxa de dividendo no momento $\mathrm{t}$;

DPAt $=$ dividendo pago por ação no período t;

$\mathrm{Pt}=$ preço da ação no momento $\mathrm{t}$.

Desse modo, a taxa de dividendo traduz para o preço das ações a expectativa de retorno do investimento, revelando rapidamente políticas passadas e esperadas de distribuição de dividendos. Uma das desvantagens para sua adequada aplicação é que políticas diferenciadas de distribuição de dividendos (taxa payout) podem gerar diferentes valores encontrados na análise das empresas (DAMODARAN, 2007).

\subsection{Trabalhos empíricos anteriores}

A questão da relevância ou não da distribuição dos dividendos a fim de gerar valor para os acionistas e a capacidade de previsão do múltiplo taxa de dividendo sobre o preço e dividendo futuro dos ativos é altamente polêmica.

Graham, Dodd e Cottle (1962) defendem a distribuição de dividendos como um meio de aumentar o retorno dos acionistas. Segundo os autores, se duas companhias com o mesmo poder de geração de lucro e posição no setor possuírem dividendos diferentes, a empresa que paga mais dividendos terá maior valor. Modigliani e Miller (1961) mostram que a política de distribuição de dividendos é indiferente para os preços das ações, sendo o valor da empresa medido pelo valor presente dos fluxos futuros do seu investimento não importando as fontes de financiamentos utilizadas. Desse modo, o estudo é sustentado na crença de que, para o investidor, é indiferente se o retorno virá na forma de dividendos ou na venda das ações. A “Teoria do Pássaro na Mão", encontrada nos trabalhos de Gordon (1963) e Walter (1963), traz uma visão diferente: na sua fundamentação os dividendos são muito mais estáveis que os preços dos ativos, assim, os investidores preferem ações que pagam dividendos no lugar das que os retêm, devido a uma menor percepção de risco, aumentando o valor das ações boas pagadoras de dividendos. 
De maneira oposta, a "Teoria da Preferência Tributária" argumenta que, caso exista uma tributação inferior ou igual aos ganhos de capital em relação aos dividendos, os investidores irão ter preferência por ações que não paguem dividendos, pois o importante é o retorno após o pagamento dos impostos (SPERANZINI, 1994). Miller e Scholes (1978) defendem o modelo clientela e irrelevância. Através do estudo das mudanças tributárias ocorridas nos USA em 1976, verificou-se a possibilidade de redução da tributação sobre os dividendos de maneira parcial ou completa por meio da dedução das despesas de juros, tornando indiferente para o investidor obter ganho com a venda da ação ou com dividendo. Shefrin e Statman (1984) analisaram a razão de haver preferência dos investidores em receber dividendo. As pesquisas mostraram que os acionistas limitam seus gastos de acordo com os dividendos recebidos; dessa maneira, cobram das empresas um pagamento constante de dividendos e não estão dispostos a vender suas ações, levando as empresas, algumas vezes, a se endividarem para manterem o pagamento dos dividendos.

A partir das divergentes teorias expostas acima, vários trabalhos foram produzidos tentando estudar a relação entre o retorno de uma aplicação e a taxa de dividendo, e seu poder de previsão. Hess (1982) analisou a relação entre a taxa de dividendo e o retorno das ações pelo efeito clientela e informacional, obtendo resultados inconclusivos. Kein (1985) verificou se os retornos estimados pela taxa de dividendo são efeitos anormais ou ligados a impostos. Foi encontrada uma relação não-linear entre as variáveis, porém, os resultados somente foram significativos com a permanência do mês de janeiro na amostra. Fama e French (1988) constataram que o poder de previsão da taxa de dividendo sobre o retorno aumenta com o tempo. Campbell e Shiller (1998, 2001) encontraram evidências de que a taxa de dividendo explica melhor a variação dos preços que o crescimento dos dividendos no mercado americano, adotando como intervalo de tempo o período necessário para a taxa de dividendo retornar a média e 10 anos.

Em estudos mais recentes, Cochrane (2008) analisou o poder de previsão da taxa de dividendo em relação ao dividendo futuro e não encontrou nenhuma relação entre as variáveis, o que significa que alterações na taxa de dividendo estão relacionadas a expectativas de mudanças no retorno futuro dos ativos, e não à variação nos dividendos subseqüentes. Já Engsted e Pedersen (2010) estudaram o poder de previsão da taxa de dividendo nos Estados Unidos, Reino Unido, Suíça e Dinamarca, concluíram que na Suíça e Dinamarca o crescimento do dividendo é altamente previsível; nos Estados Unidos o poder de previsão, de longo prazo, está relacionado ao fato de o cálculo do retorno sobre os ativos e variações no crescimento dos dividendos ter sido realizado em taxa real ou nominal. Menzly (2004) mostrou que a mudança na preferência pelo risco reduz o poder preditivo da taxa de dividendo em relação ao dividendo futuro por ação. Campbell (2003) encontrou sinais do poder de previsão em relação ao dividendo por ação no curto prazo em vários países, e Chen (2009) mostrou que, nos Estados Unidos, a taxa de dividendo permitiu prever o crescimento dos dividendos por ação até o final da $2^{\circ}$ Guerra Mundial e que, após esse período, o poder de previsão direcionou-se para o retorno.

Speranzini (1994), analisando o mercado acionário brasileiro, avaliou se o retorno esperado de uma ação é função do seu yield e risco sistemático no período de janeiro de 1985 a dezembro de 1989, buscando identificar se o acionista exigia retornos de acordo com a política de dividendos adotada. Os resultados mostraram que os dividendos eram indesejáveis, fato que foi explicado pelas regras tributárias da época que isentavam de tributação os ganhos de capital. Bueno (2002) analisou no mercado acionário brasileiro se empresas com altas taxas de dividendos são seguidas por retornos 
anormais no período entre junho de 1994 e dezembro de 1999 e a eficiência da estratégia de comprar ações com alta taxa de dividendo. $\mathrm{O}$ estudo foi realizado por meio da constituição de três carteiras separadas de acordo com a taxa de dividendo dos ativos, e os resultados não encontraram sinais de que ações com altas taxas de dividendo tendam a apresentar melhores taxas de retorno do que as ações de baixa ou zero taxa de dividendo, sinalizando uma visível falta de associação entre o retorno futuro e o valor do múltiplo taxa de dividendo.

Por outro lado, Neto e Saito (2002) encontraram uma relação direta entre a taxa de dividendo e o retorno anormal acumulado no período pós-pagamento dos dividendos. Os autores encontraram um retorno anormal acumulado nos 90 dias posteriores ao evento: de $21,97 \%$ para empresas que pagaram maiores dividendos, de $5,16 \%$ para as que pagaram dividendos intermediários e de - 15,50\% para as que pagaram dividendos mais baixos, confirmando persistência de retornos anormais no período pós-evento.

Conforme constatado, não existe consenso na literatura sobre a capacidade de previsão da taxa de dividendo e se o ajuste, caso exista, ocorre no lucro por ação ou no preço do ativo futuro. Desse modo, o estudo busca trazer novas evidências que possam contribuir para responder a tais questionamentos.

\section{METODOLOGIA}

\subsection{Cálculo da Variação e Estatística Descritiva}

A partir dos dados obtidos do dividendo por ação da carteira, no período analisado, do índice Bovespa e do número de pontos de fechamento no último dia útil do mês de junho e dezembro, foram calculadas as variações do dividendo por ação $(\Delta d)$ e do índice Bovespa $(\Delta r)$.

Seguindo trabalho de Engsted e Pedersen (2010), foram realizados cálculos em taxas reais e nominais, procurando verificar a influência da inflação na previsibilidade da taxa de dividendo. Os cálculos das respectivas variações do dividendo futuro e do retorno do índice Bovespa foram realizados pelas seguintes equações:

$$
\begin{aligned}
& \Delta \mathrm{d}_{\mathrm{t}+1}=\mathrm{d}_{\mathrm{t}+1}-\mathrm{d}_{\mathrm{t}}=\ln \left(\frac{\mathrm{d}_{\mathrm{t}+1}}{\mathrm{~d}_{\mathrm{t}}}\right)= \\
& \ln \left(1+\frac{\mathrm{d}_{\mathrm{t}+1}-\mathrm{d}_{\mathrm{t}}}{\mathrm{d}_{\mathrm{t}}}\right) \\
& \Delta \mathrm{r}_{\mathrm{t}+1}=\mathrm{p}_{\mathrm{t}+1}-\mathrm{p}_{\mathrm{t}}=\ln \left(\frac{\mathrm{p}_{\mathrm{t}+1}}{\mathrm{p}_{\mathrm{t}}}\right)= \\
& \ln \left(1+\frac{\mathrm{p}_{\mathrm{t}+1}-\mathrm{p}_{\mathrm{t}}}{\mathrm{p}_{\mathrm{t}}}\right)
\end{aligned}
$$

Sendo:

$\Delta \mathrm{d}_{\mathrm{t}+1}=$ taxa de variação do dividendo da carteira do índice Bovespa no período $\mathrm{t}+1$;

$\mathrm{d}_{\mathrm{t}+1}=$ dividendo da carteira do índice Bovespa em $\mathrm{t}+1$;

$\mathrm{d}_{\mathrm{t}}=$ dividendo da carteira do índice Bovespa em $\mathrm{t}$;

$\Delta \mathrm{r}_{\mathrm{t}+1}=$ taxa de variação da carteira do índice Bovespa no período $\mathrm{t}+1$; 
$\mathrm{p}_{\mathrm{t}+1}=$ pontos da carteira do índice Bovespa considerando fechamento em $\mathrm{t}+1$;

$\mathrm{p}_{\mathrm{t}}=$ pontos da carteira do índice Bovespa considerando fechamento em $\mathrm{t}$.

Para o cálculo da taxa real foi utilizado o Índice Nacional de Preço ao Consumidor Amplo (IPCA) fornecido pelo Instituto Brasileiro de Geografia e Estatística.

Deste modo, foi realizada inicialmente uma análise estatística descritiva das séries, sendo apresentados os cálculos da: média, mediana, desvio-padrão, máximo, mínimo, curtose e assimetria.

\subsection{Teste de Estacionaridade e Vetores Auto-Regressivos - VAR}

De modo a verificar se as séries são estacionárias foi utilizado o método de Phillips-Perron (PP). O teste de Phillips-Perron assume como hipótese nula que a série possui raiz unitária, podendo ser estimado por mínimos quadrados ordinários.

O modelo vetor auto-regressivo (VAR) é um sistema de modelos de regressões que pode ser considerado como uma espécie de híbrido entre modelos univariados de séries temporais e modelos de equações simultâneas (BROOKS, 2008). Desse modo, os modelos auto-regressivos fornecem importantes informações de causalidades entre as variáveis, decomposição da variância dos resíduos e a função de impulso-resposta, o que garante meios de entender as inter-relações entre as variáveis.

O modelo bivariado é estimado conforme equações 4.1 e 4.2

$$
\begin{aligned}
& y_{1 t}=\beta_{10}+\beta_{11} y_{1 t-1}+\ldots+\beta_{1 k} y_{1 t-k}+\alpha_{11} y_{2 t-1}+\ldots+\alpha_{1 k} y_{2 t-k}+u_{1 t} \\
& y_{2 t}=\beta_{20}+\beta_{21} y_{2 t-1}+\ldots+\beta_{2 k} y_{2 t-k}+\alpha_{21} y_{1 t-1}+\ldots+\alpha_{2 k} y_{1 t-k}+u_{2 t}
\end{aligned}
$$

Assim, as variáveis $y_{1 t}$ e $y_{2 t}$ dependem de diferentes combinações $\mathrm{K}$ anteriores do valor de ambas as variáveis e do termo de erro. É importante ressaltar que o erro é um ruído branco com $\mathrm{E}\left(u_{i t}\right)=0$ para $(i=1,2)$ e $\mathrm{E}\left(u_{1 t} u_{2 t}\right)=0$.

Um dos maiores desafios encontrados durante a modelagem por meio do sistema VAR é determinar o número de defasagens a ser utilizado. A técnica utilizada é a conhecida como "critério de informação", a qual engloba dois fatores: um termo que é função da soma dos resíduos ao quadrado e uma penalidade devido à perda de graus de liberdade com a adoção de um novo parâmetro. Desse modo, o número de parâmetros utilizado será aquele que reduzir o critério de informação.

O critério de informação é calculado pelos métodos: Akaike's (AIC), Schwarz's (SBIC) e Hannan-Quinn (HQIC). As fórmulas são apresentadas nas equações 5, 6 e 7.

$$
\begin{aligned}
& A I C=\log |\hat{\Sigma}|+\frac{2 k^{s}}{T} \\
& S B I C=\log |\widehat{\Sigma}|+\frac{k^{s}}{T} \log (T) \\
& H Q I C=\log |\widehat{\Sigma}|+\frac{2 k^{s}}{T} \log (\log ((T))
\end{aligned}
$$


Sendo:

$\widehat{\Sigma}=$ matriz de variância-covariância dos resíduos;

$\mathrm{T}$ = número de observações;

$k^{x}=$ total de número de regressores na equação.

Podemos observar pelas fórmulas 5, 6 e 7 que o método SBIC penaliza de maneira mais acentuada a perda de graus de liberdade que o método AIC, já a penalização pelo método HQIC está em algum ponto entre os métodos anteriores. Segundo Brooks (2008) não existe método superior, o SBIC é muito consistente, porém é ineficiente; o AIC não é consistente, mas geralmente é mais eficiente. Greene (2007) argumenta que o método de SBIC, devido à maior penalização pela perda de graus de liberdade, fornece modelos mais parcimoniosos. Emiliano (2009) via simulação de Monte Carlo realizou um estudo comparativo entre os métodos SBIC e AIC, para diferentes tamanhos de amostras, os resultados encontrados mostraram que o método SBIC é superior ao AIC para grandes amostras. Assim, devido a melhores propriedades assintóticas e a seleção de modelos mais parcimoniosos o método de SBIC será utilizado, como primeira opção, para a escolha do número de defasagens, caso ocorra divergências entre os métodos.

Outra dificuldade encontrada na interpretação dos modelos VAR está relacionada à causalidade entre as variáveis, ou seja, mudanças em $y_{1 t}$ causam variações em $y_{2 t}$ e vice versa. Para responder a tal questionamento será utilizado o teste de causalidade de Granger, sendo a hipótese nula a ausência de causalidade entre as variáveis.

Brooks (2008), ressalta que o teste de causalidade não revela se mudanças em uma variável têm efeitos positivos ou negativos nas outras variáveis do sistema e o tempo de duração desses efeitos. Assim, para uma correta interpretação do modelo VAR é fundamental uma análise desses efeitos, sendo utilizada a função impulso-resposta para verificar a resposta na variável dependente, devido ao choque em cada variável; e a decomposição da variância para explicar a proporção do movimento na variável dependente devido ao choque nela mesma e em outras variáveis.

Por fim, para verificar se o modelo não viola os pressupostos básicos é realizado teste de normalidade de Anderson-Darling nos resíduos.

\subsection{Fonte de dados e composição da amostra}

Os dados foram obtidos do sistema economatica, sendo ajustados por proventos, em moeda original e corrigidos pela inflação. As carteiras teóricas do Ibovespa foram coletadas no website: www.bmfbovespa.com.br.

O cálculo do dividendo por ação foi feito pela soma do dividendo com os juros sobre capital próprio. Para as empresas que não forneceram dados sobre o dividendo pago por ação e taxa de dividendo, no período, foram realizados os seguintes procedimentos:

- Aproximação do dividendo por ação através da análise do lucro líquido informado no período em conjunto com a taxa histórica de payout praticada e política de dividendos encontrada no Estatuto Social da empresa; 
- Cálculo da taxa de dividendo por meio da divisão do dividendo por ação estimado pelo preço de fechamento da ação no último dia útil do mês de junho $\left(1^{\circ}\right.$ semestre $)$ e dezembro $\left(2^{\circ}\right.$ semestre $)$;

- Caso nenhum desses dados não tenham sidos divulgados pela empresa ou ocorreu prejuízo no período foi atribuído valor zero para o dividendo por ação e taxa dividendo.

A partir da coleta da composição das carteiras teóricas quadrimestrais do índice Ibovespa, foi calculado o dividendo por ação e a taxa de dividendo para cada carteira utilizada no estudo por meio da média ponderada do dividendo por ação e taxa de dividendo de cada ação que compõe o índice Ibovespa com os seus respectivos pesos originais, conforme exemplo na Tabela 1 referente à carteira teórica utilizada no primeiro semestre de 2010.

TABELA 1

Ilustração do cálculo do dividendo por ação e taxa de dividendo do índice Bovespa para o $1^{\circ}$ semestre 2010

DPA: dividendo por ação, DY: taxa de dividendo, IBOV (\%): peso da ação na carteira teórica do índice Bovespa, DPA Ibovespa: dividendo por ação multiplicado pelo respectivo peso da empresa na carteira teórica do Ibovespa no período, DY Ibovespa: taxa de dividendo por ação multiplicado pelo respectivo peso da empresa na carteira teórica do Ibovespa no período

\begin{tabular}{|c|c|c|c|c|c|c|}
\hline Empresa & Classe & DPA & DY & IBOV (\%) & DPA Ibovespa & DY Ibovespa \\
\hline AGRE EMP IMO & ON & 0,02 & 0,27 & 1,03 & 0,02 & 0,28 \\
ALL AMER LAT & UNT & 0,00 & 0,07 & 1,38 & 0,00 & 0,10 \\
AMBEV & PN & 7,24 & 3,97 & 0,83 & 6,01 & 3,30 \\
B2W VAREJO & ON & 0,11 & 0,35 & 0,70 & 0,07 & 0,24 \\
BMF Bovespa & ON & 0,42 & 3,60 & 4,21 & 1,78 & 15,16 \\
BRADESCO & PN & 0,96 & 3,72 & 3,10 & 2,98 & 11,53 \\
BRADESPAR & PN & 1,06 & 3,20 & 0,92 & 0,98 & 2,94 \\
BRASIL & ON & 1,80 & 7,20 & 1,98 & 3,56 & 14,25 \\
BRASIL TELEC & PN & 0,00 & 0,00 & 0,41 & 0,00 & 0,00 \\
BRASKEM & PN & 0,00 & 0,00 & 0,57 & 0,00 & 0,00 \\
BRF FOODS & ON & 0,18 & 0,76 & 1,87 & 0,34 & 1,42 \\
CCR RODOVIAS & ON & 1,46 & 3,87 & 0,72 & 1,05 & 2,78 \\
CEMIG & PN & 1,40 & 5,27 & 1,35 & 1,88 & 7,11 \\
CESP & PNB & 0,41 & 1,63 & 0,80 & 0,33 & 1,31
\end{tabular}

Continuação

\begin{tabular}{|c|c|c|c|c|c|c|}
\hline Empresa & Classe & DPA & DY & IBOV (\%) & DPA Ibovespa & DY Ibovespa \\
\hline CIELO & ON & 0,80 & 5,19 & 1,98 & 1,58 & 10,27 \\
COPEL & PNB & 1,00 & 2,65 & 0,61 & 0,61 & 1,62 \\
COSAN & ON & 0,00 & 0,00 & 0,77 & 0,00 & 0,00 \\
CPFL ENERGIA & ON & 2,66 & 6,68 & 0,50 & 1,33 & 3,34 \\
Cyrela Realty & ON & 0,48 & 2,42 & 1,77 & 0,85 & 4,29 \\
DURATEX & ON & 0,08 & 0,50 & 0,59 & 0,05 & 0,29 \\
ECODIESEL & ON & 0,00 & 0,00 & 0,94 & 0,00 & 0,00 \\
ELETROBRAS & PNB & 1,89 & 6,63 & 0,83 & 1,57 & 5,50 \\
ELETROBRAS & ON & 12,20 & 50,51 & 1,00 & 12,20 & 50,51 \\
ELETROPAULO & PNB* & 6,91 & 18,97 & 0,67 & 4,63 & 12,71 \\
EMBRAER & ON & 0,38 & 4,02 & 0,85 & 0,32 & 3,42
\end{tabular}




\begin{tabular}{|c|c|c|c|c|c|c|}
\hline FIBRIA & ON & 0,00 & 0,00 & 2,03 & 0,00 & 0,00 \\
\hline GAFISA & ON & 0,12 & 1,13 & 1,63 & 0,20 & 1,85 \\
\hline GERDAU & PN & 0,39 & 1,63 & 3,43 & 1,34 & 5,59 \\
\hline GERDAU MET & PN & 0,61 & 2,06 & 0,88 & 0,54 & 1,81 \\
\hline GOL & PN & 0,72 & 3,33 & 1,03 & 0,74 & 3,43 \\
\hline ITAUSA & PN & 0,34 & 3,09 & 2,45 & 0,82 & 7,58 \\
\hline ITAUUNIBANCO & PN & 0,91 & 2,77 & 3,87 & 3,53 & 10,73 \\
\hline JBS & ON & 0,03 & 0,35 & 0,97 & 0,03 & 0,34 \\
\hline KLABIN S/A & PN & 0,18 & 3,48 & 0,45 & 0,08 & 1,57 \\
\hline LIGHT S/A & ON & 2,66 & 12,51 & 0,54 & 1,44 & 6,76 \\
\hline LLX LOG & ON & 0,00 & 0,00 & 0,95 & 0,00 & 0,00 \\
\hline LOJAS AMERIC & PN & 0,05 & 0,41 & 1,06 & 0,06 & 0,44 \\
\hline LOJAS RENNER & ON & 1,20 & 2,42 & 1,01 & 1,21 & 2,45 \\
\hline MMX MINER & ON & 0,00 & 0,00 & 1,23 & 0,00 & 0,00 \\
\hline MRV & ON & 0,17 & 1,35 & 0,97 & 0,17 & 1,31 \\
\hline NATURA & ON & 1,45 & 3,57 & 0,78 & 1,13 & 2,79 \\
\hline NET & PN & 0,00 & 0,00 & 0,88 & 0,00 & 0,00 \\
\hline OGX PETROLEO & ON & 0,00 & 0,00 & 2,42 & 0,00 & 0,00 \\
\hline P.Acucar-Cbd & PN & 0,70 & 1,09 & 0,72 & 0,50 & 0,79 \\
\hline PDG REALT & ON & 0,11 & 1,37 & 1,10 & 0,12 & 1,50 \\
\hline PETROBRAS & ON & 1,20 & 3,83 & 2,74 & 3,29 & 10,49 \\
\hline PETROBRAS & PN & 1,20 & 4,42 & 10,63 & 12,77 & 46,96 \\
\hline REDECARD & ON & 2,16 & 8,35 & 1,71 & 3,69 & 14,28 \\
\hline ROSSI RESID & ON & 0,20 & 1,50 & 1,03 & 0,20 & 1,55 \\
\hline SABESP & ON & 1,17 & 3,09 & 0,35 & 0,41 & 1,08 \\
\hline SID NACIONAL & ON & 1,28 & 4,79 & 2,49 & 3,18 & 11,93 \\
\hline SOUZA CRUZ & ON & 4,96 & 7,20 & 0,47 & 2,33 & 3,39 \\
\hline TAM S/A & PN & 1,61 & 6,35 & 0,88 & 1,41 & 5,59 \\
\hline TELEMAR & ON & 3,21 & 8,40 & 0,24 & 0,77 & 2,02 \\
\hline TELEMAR & PN & 3,21 & 11,73 & 0,93 & 2,98 & 10,91 \\
\hline TELEMAR N L & PN & 0,00 & 0,00 & 0,25 & 0,00 & 0,00 \\
\hline TELESP & PN & 3,91 & 10,69 & 0,17 & 0,66 & 1,82 \\
\hline TIM PART S/A & ON & 0,00 & 0,00 & 0,14 & 0,00 & 0,00 \\
\hline Continuação & & & & & & \\
\hline Empresa & Classe & DPA & DY & IBOV (\%) & DPA Ibovespa & DY Ibovespa \\
\hline TIM PART S/A & $\mathrm{PN}$ & 0,13 & 2,61 & 0,82 & 0,10 & 2,14 \\
\hline TRAN PAULIST & PN & 4,95 & 10,52 & 0,26 & 1,29 & 2,74 \\
\hline ULTRAPAR & PN & 2,17 & 2,48 & 0,50 & 1,08 & 1,24 \\
\hline USIMINAS & ON & 0,48 & 1,98 & 0,67 & 0,32 & 1,33 \\
\hline USIMINAS & PN & 0,52 & 2,15 & 2,88 & 1,51 & 6,18 \\
\hline VALE & ON & 0,95 & 2,15 & 2,74 & 2,60 & 5,89 \\
\hline VALE & PN & 0,95 & 2,48 & 10,63 & 10,10 & 26,32 \\
\hline VIVO & PN & 2,15 & 4,58 & 0,70 & 1,51 & 3,20 \\
\hline \multicolumn{5}{|c|}{ MÉDIA PONDERADA } & 1,04 & 3,60 \\
\hline
\end{tabular}

Fonte: Elaboração do autor 
Foram construídas no total 34 carteiras semestrais, no período entre 1994 a 2010, sendo utilizado o dividendo por ação e a taxa de dividendo do último dia útil do mês de junho e dezembro das empresas que compunham a carteira teórica do Ibovespa do $2^{\circ}$ e $3^{\circ}$ quadrimestres, respectivamente, mantendo seus pesos originais para refletir da maneira mais próxima possível o dividendo por ação e a taxa de dividendo da carteira teórica do Bovespa.

\section{APRESENTAÇÃO E ANÁLISE DOS DADOS}

\subsection{Estatísticas Descritivas}

Inicia-se a apresentação dos dados por meio da estatística descritiva das séries durante o período de 1994 a 2010, com as respectivas médias, medianas, desviospadrões, mínimos, máximos, curtoses, assimetrias e testes de normalidade de AndersonDarling, conforme Tabela 2:

Tabela 2

Estatística descritiva dos dados no período de 1994 a 2010

DY - taxa de dividendo, $\Delta$ d_orig - variação do dividendo da carteira do Ibovespa em moeda original, $\Delta \mathrm{r}$ _orig - retorno do índice em moeda original, $\Delta \mathrm{d}$ inf - variação do dividendo da carteira Ibovespa ajustado pela inflação, $\Delta \mathrm{r}$ inf - retorno do índice ajustado pela inflação. Dados percentuais.

\begin{tabular}{|c|c|c|c|c|c|c|c|c|}
\hline Variável & Média & Mediana & Desvio-padrão & Mínimo & Máximo & Curtose & Assimetria & Valor-p* \\
\hline DY & 3,526 & 3,363 & 1,413 & 0,689 & 6,764 & 2,921 & 0,180 & 0.813 \\
$\Delta$ d_orig & 11,576 & 3,838 & 38,486 & $-70,790$ & 125,790 & 4,280 & 0,780 & 0.054 \\
$\Delta$ r_orig & 8,953 & 13,191 & 25,307 & $-54,900$ & 57,950 & 3,053 & $-0,152$ & 0.723 \\
$\Delta$ d_inf & 2,980 & 0,909 & 30,325 & $-63,950$ & 80,190 & 3,367 & 0,337 & 0.659 \\
$\Delta$ r_inf & 5,009 & 7,541 & 25,257 & $-57,050$ & 53,940 & 2,945 & $-0,076$ & 0.835 \\
\hline
\end{tabular}

Notas:

* Valor -p do teste de normalidade Anderson-Darling.

Fonte: Elaboração autor, a partir dados analisados no software Stata e Minitab.

Observando a Tabela 2 verifica-se que a taxa de dividendo (DY) média no período foi $3,526 \%$. Ocorreu grande diferença entre a variação média do dividendo da carteira do Ibovespa em dados originais ( $\Delta$ d_orig) e ajustados pela inflação $(\Delta d$ _inf), $11,576 \%$ contra $2,98 \%$, respectivamente, tornando evidente a grande influência da inflação no período. Outro ponto relevante é a grande dispersão dos dados, principalmente a da série variação do dividendo por ação da carteira do Ibovespa em moeda original $(\Delta \mathrm{d}$ _orig) que apresentou um desvio-padrão de $38,486 \%$, máximo de $125,79 \%$ e mínimo de $-70,79 \%$.

Todas as séries apresentaram curtose próxima de 3, com exceção da série de dividendo por ação em moeda original. $\mathrm{O}$ teste de normalidade de Anderson-Darling não rejeitou a hipótese nula de distribuição normal em nenhuma das séries analisadas, a $5 \%$ de significância.

O Gráfico 1 apresenta a variação da taxa de dividendo (DY), o retorno (Var Ibov) e a taxa de variação do dividendo por ação (Var DPA), da série semestral, formada a partir da carteira do Ibovespa no período de 1994 a 2010, em taxa real (inf.) e nominal (orig.). 


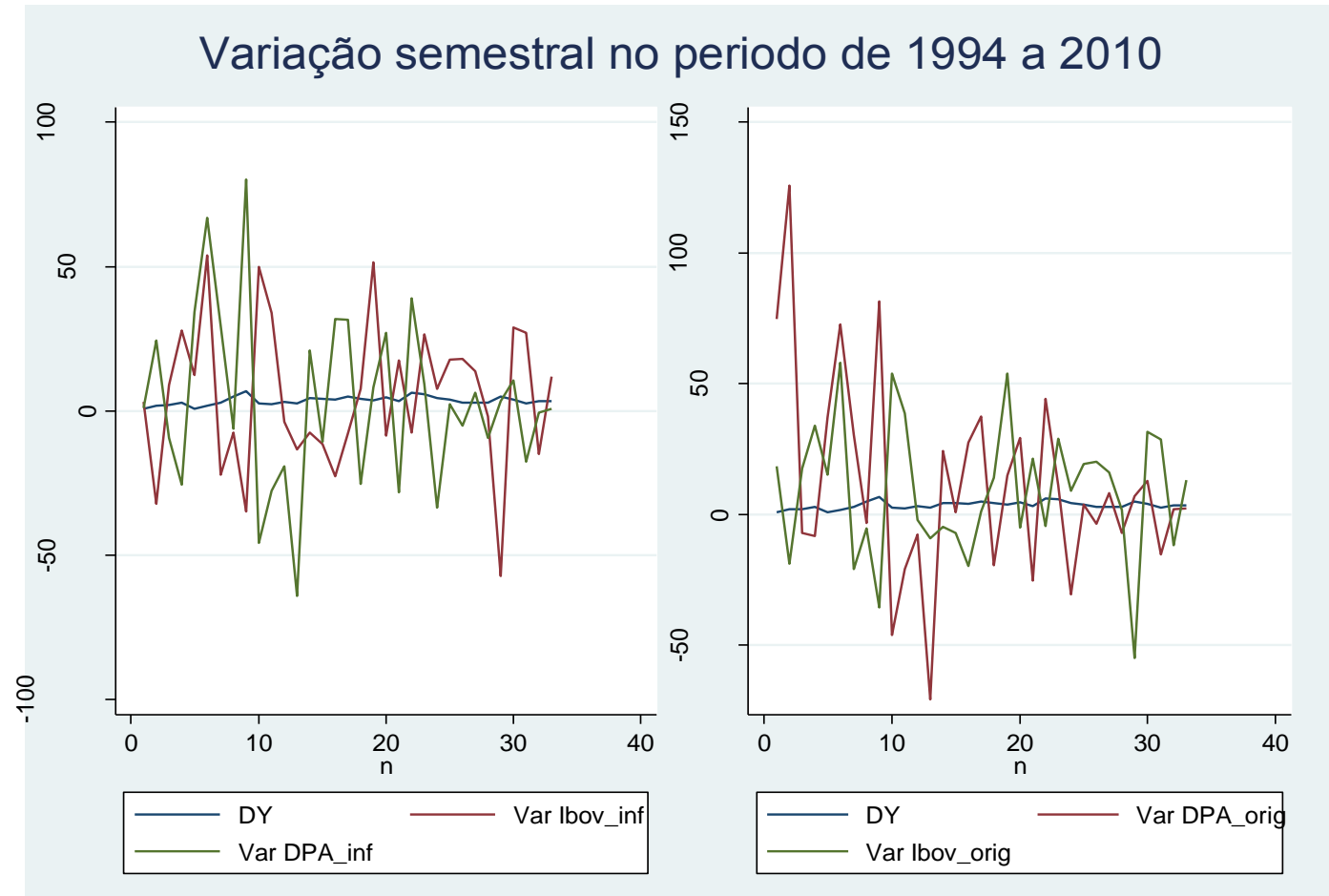

Gráfico 1: Variação das séries no período 1994 a 2010.

Fonte: Elaboração autor, a partir dados analisados no software Stata 10.

Pelo gráfico, há evidências da presença de clusters de volatilidade na série da variação do dividendo por ação e do retorno da carteira do Ibovespa, em moeda original e ajustado por inflação. Outro ponto relevante são sinais de presença de dados atípicos na série da variação do dividendo por ação da carteira do Ibovespa e sinais de estacionaridade em todas as séries.

\subsection{Análise de estacionaridade e estimação pelo modelo de vetores auto-regressivos} VAR

A Tabela 3 apresenta o teste de estacionaridade das séries, utilizando o teste de Phillips-Perron.

Tabela 3

Teste de estacionaridade de Phillips-Perron

DY - taxa de dividendo, $\Delta$ d_orig - variação do dividendo da carteira do Ibovespa em moeda original, $\Delta \mathrm{r}$ orig - retorno do índice em moeda original, $\Delta \mathrm{d}$ inf - variação do dividendo da carteira Ibovespa ajustado pela inflação, $\Delta \mathrm{r}$ _inf - retorno do índice ajustado pela inflação.

\begin{tabular}{c|cc}
\hline \multirow{2}{*}{ Série } & \multicolumn{2}{|c}{ Phillips-Perron } \\
\cline { 2 - 3 } & $\mathrm{T}$ & valor-p \\
\hline DY & $-3,685^{*}$ & 0,004 \\
$\Delta$ d_orig & $-5,300^{*}$ & 0,000 \\
$\Delta \mathrm{r}$ _orig & $-6,170^{*}$ & 0,000 \\
$\Delta \mathrm{d}$ _inf & $-5,867^{*}$ & 0,000 \\
$\Delta \mathrm{r}$ inf & $-5,948^{*}$ & 0,000 \\
\hline
\end{tabular}

Notas:

Valores críticos para o teste de Phillips-Perron -3,702 (1\%), -2,980 (5\%) e -2,662 (10\%). * significante a $5 \%$.

Fonte: Elaboração autor, a partir dados analisados no software Stata 10.

Observa-se que as séries são estacionárias pelo teste de Phillips-Perron. 


\subsection{Modelos de VAR em moeda original}

\subsubsection{Estimação com as variáveis taxa de dividendo e dividendo por ação do Ibovespa}

Os testes de Akaike's (AIC), Schwarz's (SBIC) e Hannan-Quinn (HQIC), para determinar o número de defasagens, são mostrados na Tabela 4.

Tabela 4

Teste modelo VAR do dividendo por ação em moeda original

\begin{tabular}{c|ccc|}
\hline Defasagem & AIC & HQIC & SBIC \\
\hline 0 & 13,153 & 13,183 & 13,248 \\
1 & 12,965 & $13,053^{*}$ & $13,247^{*}$ \\
2 & 13,121 & 13,269 & 13,592 \\
3 & 13,057 & 13,263 & 13,761 \\
4 & $12,811^{*}$ & 13,077 & 13,660 \\
\hline
\end{tabular}

Nota:

* Os valores com asteriscos representam o número de defasagem a ser utilizado no modelo VAR de acordo com respectivo critério de seleção.

Fonte: Elaboração autor, a partir dados analisados no software Stata 10.

De acordo com o critério HQIC e SBIC o número de defasagens a ser utilizado é um, resultado distinto do método AIC. Desse modo, o modelo será estimado com uma defasagem.

A Tabela 5 apresenta as estimativas do modelo (painel a), o teste de causalidade de Granger (painel b) e teste de normalidade dos resíduos (painel c).

Tabela 5

Modelo VAR do dividendo por ação em moeda original, teste causalidade e de normalidade dos resíduos

$\Delta$ d_orig - variação do dividendo da carteira do Ibovespa em moeda original, DY - taxa de dividendo.

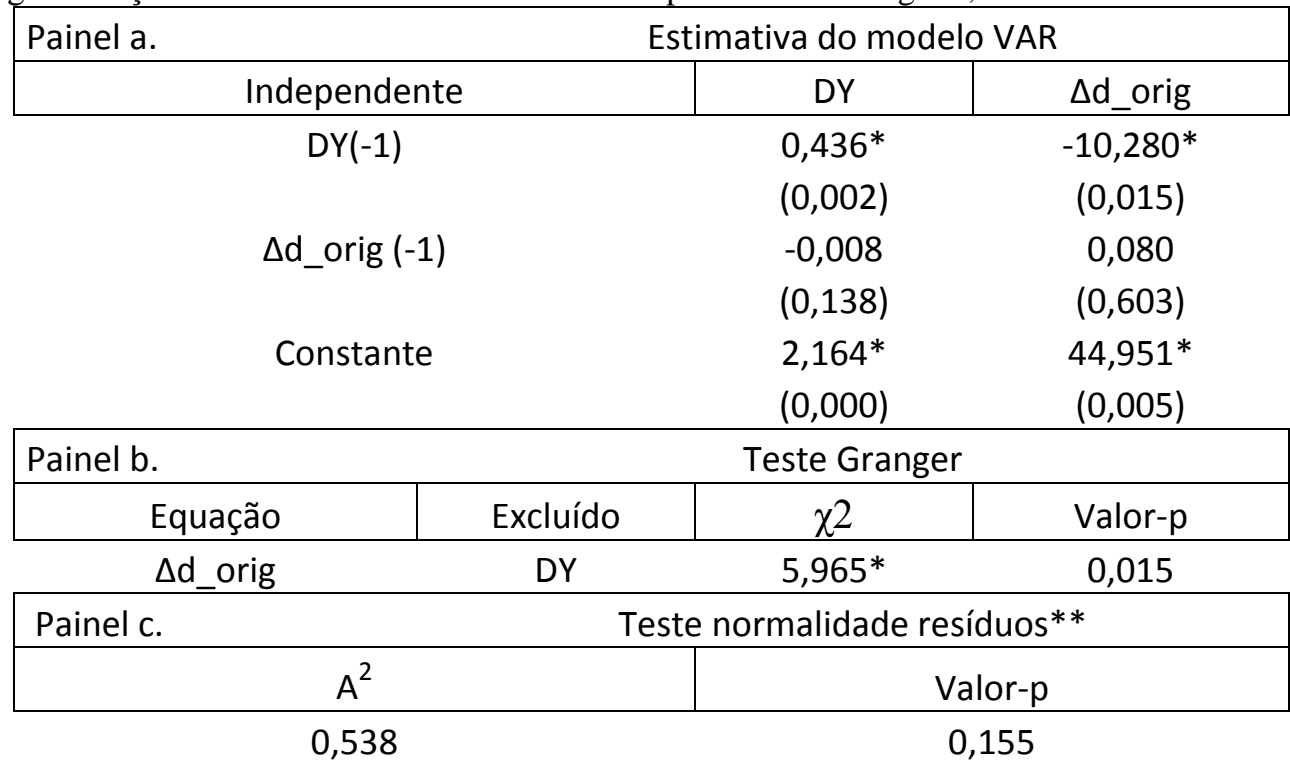

Notas:

( ) Os valores entre parênteses representam os p-values dos coeficientes.

* Significante a $5 \%$.

** Teste de normalidade Anderson-Darling.

Fonte: Elaboração autor, a partir dados analisados no software Stata 10 e Minitab. 
Constata-se que a variável taxa de dividendo é significante para explicar a variação do dividendo por ação da carteira do Ibovespa um semestre à frente, a estimativa do coeficiente xx1 da equação yy1 é de -10,280 e valor-p de 0,015; a constante também é significativa (p-valor de 0,005).

$\mathrm{O}$ teste de causalidade de Granger rejeita hipótese nula, ou seja, variações defasadas na taxa de dividendo geram melhores previsões estatisticamente nas variações no dividendo por ação; os resíduos têm distribuição normal.

Desse modo, a variação do dividendo por ação da carteira do Ibovespa pode ser estimada pela equação abaixo:

$$
\begin{gathered}
\Delta \mathrm{d}_{\text {_orig }}=-10,280 . \mathrm{DY}(-1)+0,080 . \Delta \mathrm{d}_{\text {-orig }}(-1) \\
+44,951
\end{gathered}
$$

A função impulso-resposta mostra que um impulso dado positivamente na variação da taxa de dividendo provoca de forma complementar uma queda no dividendo por ação da carteira do Ibovespa, o que dura por 4 semestres após o impacto. A partir desse ponto, há convergência e dissipação do efeito do impacto.

Assim, a compra de ações com alta taxa de dividendo não gera retornos anormais, dado que o ajuste ocorre através da redução do dividendo por ação futuro, como esperado pela Hipótese do Mercado Eficiente (FAMA, 1970), e não por meio da valorização dos ativos

Por fim, o teste de decomposição de variância mostra que a proporção do movimento da variável dependente, "variação do dividendo por ação da carteira do Ibovespa", é maior quando ocorre choque nela mesma, em torno de $80 \%$. Depois de três semestres estabiliza-se e mantém-se em torno de $70 \%$. A influência da taxa de dividendo é de $30 \%$ no movimento da variável dependente.

\subsubsection{Estimação com as variáveis taxa de dividendo e retorno do Ibovespa}

A Tabela 6 mostra os testes de Akaike's (AIC), Schwarz's (SBIC) e HannanQuinn (HQIC) para determinar o número de defasagens usadas para estimar o modelo VAR.

Tabela 6

Teste modelo VAR do retorno do Ibovespa em moeda original

\begin{tabular}{c|c|c|c|}
\hline Defasagem & AIC & HQIC & SBIC \\
\hline 0 & 12,587 & 12,617 & $12,682^{*}$ \\
1 & $12,458^{*}$ & $12,547^{*}$ & 12,741 \\
2 & 12,480 & 12,628 & 12,952 \\
3 & 12,606 & 12,813 & 13,266 \\
4 & 12,718 & 13,017 & 13,629 \\
\hline
\end{tabular}

Nota:

* Os valores com asteriscos representam o número de defasagens a ser utilizado no modelo VAR de acordo com respectivo critério de seleção.

Fonte: Elaboração autor, a partir dados analisados no software Stata 10.

Observam-se divergências entre os critérios de informação que indicam o número de defasagens a serem utilizadas no modelo VAR. Como o critério SBIC sugere nenhuma defasagem, utilizaremos, nesse caso, como critério de escolha do número de 
defasagens o critério HQIC e AIC. A Tabela 7 apresenta os resultados com uma defasagem.

Tabela 7

Modelo VAR do retorno do Ibovespa em moeda original $\Delta \mathrm{r}$ _orig - retorno do índice em moeda original, DY - taxa de dividendo.

Notas:

\begin{tabular}{c|cc|}
\hline Independente & DY & $\Delta r$ rorig \\
\hline DY(-1) & $0,406^{*}$ & 3,242 \\
& $(0,010)$ & $(0,338)$ \\
$\Delta$ r_orig (-1) & $-0,004$ & $-0,164$ \\
& $(0,647)$ & $(0,931)$ \\
Constante & $2,213^{*}$ & $-2,644$ \\
& $(0,000)$ & $(0,884)$ \\
\hline
\end{tabular}

( ) Os valores entre parênteses representam os p-values dos coeficientes.

* Significante a $5 \%$.

Fonte: Elaboração autor, a partir dados analisados no software Stata 10.

Observa-se que a variável taxa de dividendo não explica a variação do retorno futuro do Ibovespa (valor-p de 0,338).

\subsection{Modelos de regressão em moeda ajustada pela inflação}

\subsubsection{Estimação com as variáveis taxa de dividendo e dividendo por ação do Ibovespa}

A Tabela 8 mostra o teste de Akaike's (AIC), Schwarz's (SBIC) e HannanQuinn (HQIC) para determinar o número de defasagens.

Tabela 8

Teste modelo VAR do dividendo por ação em moeda ajustada pela inflação

Nota:

\begin{tabular}{c|ccc|}
\hline Defasagem & AIC & HQIC & SBIC \\
\hline 0 & 13,216 & 13,151 & $13,216^{*}$ \\
1 & 12,980 & $13,069^{*}$ & 13,263 \\
2 & 13,160 & 13,308 & 13,632 \\
3 & 13,224 & 13,430 & 13,884 \\
4 & $12,954^{*}$ & 13,221 & 13,803 \\
\hline
\end{tabular}

* Os valores com asteriscos representam o número de defasagem a ser utilizado no modelo VAR de acordo com respectivo critério de seleção.

Fonte: Elaboração autor, a partir dados analisados no software Stata 10.

Segundo a Tabela 8, existe um grande conflito entre os critérios de seleção do número de defasagens. Como o critério de SBIC sugere nenhuma defasagem, será estimado modelo de defasagem pelo critério de HQIC com uma defasagem e com quatro defasagens segundo critério de AIC. A Tabela 9 apresenta as estimativas dos modelos com uma defasagem (painel a), quatro defasagens (painel b), teste da causalidade de Granger em modelo com quatro defasagens (painel c) e teste de normalidade dos resíduos em modelo com quatro defasagens (painel d). 
Tabela 9

Modelo VAR do dividendo por ação em moeda ajustada por inflação com 1 e 4 defasagem, teste causalidade e de normalidade dos resíduos para modelo com 4

defasagem

$\Delta$ d_inf - variação do dividendo da carteira do Ibovespa em moeda ajustada pela inflação, DY - taxa de dividendo.

\begin{tabular}{|ccc|}
\hline Painel a. & \multicolumn{3}{c|}{ Estimativa do modelo VAR com 1 defasagem } \\
\hline Independente & DY & $\Delta$ d_inf \\
\hline DY(-1) & $0,476^{*}$ & $-5,212$ \\
& $(0,001)$ & $(0,172)$ \\
$\Delta$ d_inf $(-1)$ & $-0,007$ & $-0,004$ \\
& $(0,307)$ & $(0,980)$ \\
Constante & $1,954^{*}$ & 21,459 \\
& $(0,000)$ & $(0,134)$ \\
\hline
\end{tabular}

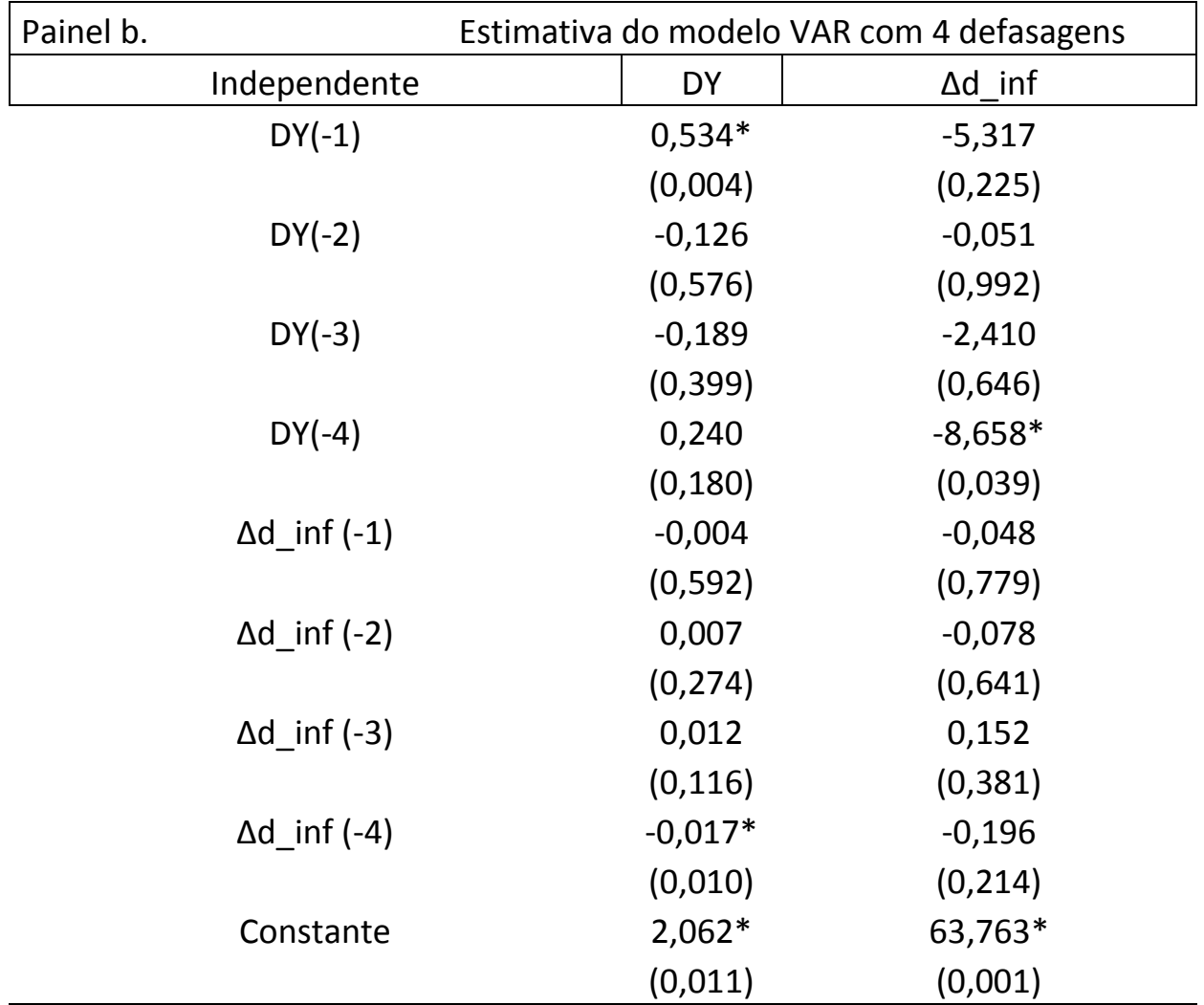

Painel c. Teste de Granger para modelo com 4 defasagens

\begin{tabular}{|c|c|c|c|}
\hline Equação & Excluído & $\chi 2$ & Valor-p \\
\hline
\end{tabular}

$\Delta$ d_inf DY $\quad$ 12,285* 0,015

\begin{tabular}{|c|c|}
\hline Painel d. & \multicolumn{2}{c|}{ Teste de normalidade para modelo com 4 defasagens** } \\
\hline $\mathrm{A}^{2}$ & Valor-p \\
\hline 0,382 & 0,376 \\
\hline
\end{tabular}

Nota:

( ) Os valores entre parênteses representam os p-values dos coeficientes.

* Significante a $5 \%$.

** Teste de normalidade Anderson-Darling.

Fonte: Elaboração autor, a partir dados analisados no software Stata 10 e Minitab. 
Constata-se que o modelo com uma defasagem não é significativo, porém, no modelo com quatro defasagens, a quarta defasagem é significativa (valor-p 0,039) tendo como variável independente o DY(-4). O teste de causalidade de Granger é significativo (valor-p de 0,015), ou seja, a variação da taxa de dividendo causa alterações no dividendo por ação ajustado por inflação.

O modelo estimado é apresentado na equação 11.

$\Delta \mathrm{d}_{\text {-inf }}=-5,317 \cdot \mathrm{DY}(-1)-0,051 \cdot \mathrm{DY}(-2)-2,410 . \mathrm{DY}(-3)-8,658 . \mathrm{DY}(-4)-0,048 . \Delta \mathrm{d}_{\text {inf }}(-1)$

$-0,078 \cdot \Delta \mathrm{d}_{\text {inf }}(-2)+0,152 \cdot \Delta \mathrm{d}_{\text {inf }}(-3)-0,196 \cdot \Delta \mathrm{d}_{\text {inf }}(-4)+63,76$

Assim, uma variação de $1 \%$ na taxa de dividendo causa uma queda de $-8,658 \%$ no dividendo quatro semestres à frente.

A função impulso resposta mostra que o impulso dado positivamente na variação da taxa de dividendo provoca de forma complementar uma queda no dividendo por ação da carteira do Ibovespa no $1^{\circ}$ semestre que é acentuada no $4^{\circ}$ semestre. A partir de 7 semestres ocorre uma inversão, aumento do dividendo. Outro ponto relevante é a não dissipação do efeito do impacto até 8 semestres. Finalizando, a decomposição da variância mostra que a proporção do movimento da variável dependente, variação do dividendo por ação da carteira do Ibovespa ajustada pela inflação, é afetada principalmente por choque nela mesma, em torno de $80 \%$. Depois do $5^{\circ}$ semestre estabiliza-se e mantém-se superior à influência do choque da taxa de dividendo, $56 \%$ e $44 \%$, respectivamente.

\subsubsection{Estimação com as variáveis taxa de dividendo e retorno do Ibovespa}

Por fim, a Tabela 10 apresenta o teste de Akaike's (AIC), Schwarz's (SBIC) e Hannan-Quinn (HQIC) para determinar o número de defasagens a ser utilizado no modelo VAR.

Tabela 10

Teste modelo VAR do retorno do Ibovespa em moeda ajustada pela inflação

\begin{tabular}{c|c|c|c|}
\hline Defasagem & AIC & HQIC & SBIC \\
\hline 0 & 12,566 & 12,595 & $12,659^{*}$ \\
1 & $12,437^{*}$ & $12,525^{*}$ & 12,720 \\
2 & 12,484 & 12,632 & 12,956 \\
3 & 12,591 & 12,798 & 13,251 \\
4 & 12,748 & 13,013 & 13,596 \\
\hline
\end{tabular}

Nota:

* Os valores com asteriscos representam o número de defasagem a ser utilizado no modelo VAR de acordo com respectivo critério de seleção.

Fonte: Elaboração autor, a partir dados analisados no software Stata 10.

Há divergência entre os critérios de informação. Como o critério SBIC não indica defasagem, será estimado um modelo de VAR com uma defasagem, conforme critério de HQIC e AIC. A Tabela 11 mostra os resultados. 
Tabela 11

Modelo VAR do retorno do Ibovespa em moeda ajustada pela inflação $\Delta r_{-}$inf - retorno do índice em moeda ajustada pela inflação, DY - taxa de dividendo.

\begin{tabular}{|c|c|c|}
\hline Independente & DY & $\Delta \mathrm{r} \_$inf \\
\hline DY(-1) & $\begin{array}{c}0,416 \\
(0,007)\end{array}$ & $\begin{array}{c}4,125 \\
(0,208)\end{array}$ \\
\hline $\begin{array}{l}\Delta r \_i n f(-1) \\
\text { continuação }\end{array}$ & $-0,003$ & 0,019 \\
\hline Independente & DY & $\Delta \mathrm{r} \_$inf \\
\hline Constante & $\begin{array}{c}(0,715) \\
2,159 \\
(0,000)\end{array}$ & $\begin{array}{l}(0,917) \\
-9,586 \\
(0,449)\end{array}$ \\
\hline
\end{tabular}

Nota:

( ) Os valores entre parênteses representam os p-values dos coeficientes.

* Significante a $5 \%$.

Fonte: Elaboração autor, a partir dados analisados no software Stata 10.

Observa-se que a variável independente taxa de dividendo é insignificante para explicar o retorno futuro do Ibovespa.

\section{CONCLUSÃO}

Embora existam várias pesquisas sobre o poder de previsão da taxa de dividendo sobre a variação futura do dividendo e retorno por ação, os trabalhos mostram que ainda estamos longe de um consenso entre os pesquisadores.

Os resultados obtidos da análise do período de 1994 a 2010, usando a carteira do Ibovespa como uma proxy do mercado, permitem extrair algumas informações importantes em relação às estratégias de investimento utilizando a taxa de dividendo na seleção de ativos.

Em primeiro lugar, existem evidências de estacionaridade nas séries taxa de dividendo, variação futura do dividendo e retorno do índice Bovespa, em moeda original e ajustada por inflação.

Os ajustes de modelos auto-regressivos (VAR), tendo como variável independente a taxa de dividendo, foram significativos somente quando utilizado como variável dependente a variação do dividendo por ação da carteira do Ibovespa, resultado contrário ao encontrado nos trabalhos de Cochrane (2008) e Campbell e Shiller (1998, 2001) em que a taxa de dividendo foi significante em prever o retorno. Em moeda original o modelo foi significante na previsão de um semestre à frente e, quando adotada moeda ajustada por inflação foi significante somente na previsão do quarto semestre. Em relação à previsão sobre o retorno do índice Bovespa, os modelos autoregressivos apresentaram resultados insignificantes, em séries originais e corrigidas pela inflação.

A diferença dos resultados obtidos entre as séries em moeda original e corrigidas por inflação, na previsão do dividendo futuro, mostra o efeito da inflação no período. Resultado semelhante foi encontrado em Engsted e Pedersen (2010), em que o poder de previsão do múltiplo estava relacionado à exclusão ou não da inflação nas séries analisadas. Segundo os autores, muitas vezes a taxa de dividendo possui o poder de prever a inflação futura e não a variação do dividendo.

Os resultados encontrados estão de acordo com a Hipótese de Eficiência do Mercado proposta por Fama (1970), uma vez que o ajuste ocorre no dividendo futuro e 
não nos preços, tornando a estratégia da taxa de dividendo como critério de seleção de ativos pouco eficiente.

Por fim, conclui-se que os resultados apresentados encontraram evidências de que a taxa de dividendo explica a variação do dividendo futuro, e é insignificante em relação à explicação do retorno do índice Bovespa. Em função das restrições impostas ao processo de seleção da amostra, recomenda-se a continuidade do presente estudo, com a ampliação do número de semestres, análise de empresas que não compõem a carteira do índice Ibovespa e análise da relação entre a taxa de dividendo e a inflação. 


\section{REFERÊNCIAS}

BM\&FBOVESPA. Índice Bovespa Definição e Metodologia. Disponível em <http://www.bmfbovespa.com.br/Indices/download/IBovespa.pdf >. Acesso em: 2 nov. 2010.

BROOKS, C. Introductory Econometrics for Finance. Cambridge: Cambridge University press, 2008.

BUENO, Arthur Franco. Os Dividendos Como Estratégias de Investimentos em Ações. Revista de Contabilidade \& Finanças-USP, São Paulo, 28, p.39-55, Jan / Abr 2002.

CAMPBELL, J.Y., SHILLER, R.J. The dividend-price ratio and expectations of future dividends and discount factors. Review of Financial Studies 1, 195-228, 1998a.

Stock Prices, Earnings, and Expected Dividends. Journal of Finance, 43(3): 661-676, July 1988(b). Valuation Ratios and the Long-Run Stock Market Outlook. The Journal of Portfolio Management, Winter, 1998, 11-26. Valuation Ratios and the Long-Run Stock Market Outlook: An Update. NBER Working Paper Series, Working Paper 8221, 2001.

CHEN, L. On the reversal of return and dividend predictability: a tale of two periods. Journal of Financial Economics 92, 128-151, 2009.

COCHRANE, J.H. The dog that did not bark. A defense of return predictability. Review of Financial Studies 21, 1533-1575, 2008.

DAMODARAN, Aswath. Avaliação de investimentos: Ferramentas e técnicas para a Determinação de qualquer ativo. Rio de Janeiro: Qualitymark, 2007.

ENGSTED, Tom; PEDERSEN, Thomas Q. The dividend-price ratio does predict dividend growth: International evidence. The Journal of Empirical Finance, v.17, pg 585- 605, 2010.

EMILIANO, Paulo César et al. Foundations and comparison of information criteria Akaike and Bayesian. Revista Brasileira de Biometria, São Paulo, v.25, n.3, p.07-21, jul.-set.2007.

FAMA, Eugene F. Efficient Capital Markets: a review of theory and empirical work. The Journal of Finance. New York: The American Finance Association, v.25, n.1 p.383-417, mar. 1970.

FONTES, R. ou FONTES, R.M.O. ; ARBEX, M. A. ; SILVA JR, G. E. . Estabilização Econômica No Brasil: Reflexões Sobre o Plano Real. Indicadores Econômicos, Porto Alegre, v. 26, n. 3, p. 73-86, 1998

GORDON, Myron J. Optimal Investment and Financing Policy. The Journal of Finance. New York: The American Finance Association, v.18, n.2, 9.264-272, mai.1963.

GRAHAN, Benjamin; DODD, David L.; COTTLE, Sidney. Security Analysis: principles and technique. 4ed. New York. McGraw-Hill, 1962.

GRASEL, Dirceu . Brasil: Plano Real e a Estabilização Econômica Inacabada. Informe Gepec, Cascavel/PR, v. 9, n. 1, p. 73-89, 2005

GREENE, W.H. Econometric analysis. $6^{\circ}$ ed. New Jersey: Hardcover, 2007

GUJARATI, Damodar N. Econometria Básica. $3^{\circ}$ ed. São Paulo: Pearson, 2004.

HESS, Patrick J. The Ex-dividend Day Behavior of Stock Returns: further evidence on tax effects. The Journal of Finance. New York: The American Finance Association, v.37, n.2, p.445-456, mai.1982.

HATANAKA, Michio. Time series based-based econometrics: unit roots and cointegration. New York: Oxford University Press, 1998. 294 p. 
HEIJ, Christiaan et al. Econometric Methods with Applications in Business and Economics. Oxford: Oxford University press, 2004.

HOLANDA, N. Introdução à Economia: da teoria à prática e da visão micro a macroperspectiva. 8 ed. Petrópolis, RJ: Vozes, 2003.

KEIM, Donald B. Dividend Yields and Stock Returns - implications of abnormal january results. Journal of Financial Economics. Amsterdam: North-Holland, v.14, n.3, p.473-489, set.1985.

MANDELBROT, B. Forecasts of future prices, unbiased markets, and Martingale models. Journal of Business, Chicago: University ofChicago, v. 39, Special Supplement, p. 242-255, Jan. 1966.

MATIAS, Alberto Borges. Análise Financeira e Fundamentalista de Empresas. São Paulo: Atlas, 2009.

MENZLY, L., SANTOS, T., VERONESI, P. Understanding predictability. Journal of Political Economy 112, 1-47, 2004.

MILLER, Merton H.; SCHOLES, Myron S. Dividends and Taxes. Journal of Financial Economics. Amsterdam: North-Holland, v.6, n.4, p.333-64, dez.1978.

MODIGLIANI, F. e MILlER, M. The Cost of Capital, Corporation Finance and the Theory of Investment. American Economic Review, v. 48, n. 3, June 1958, p.261-297.

Dividend Policy, Growth and the Valuation of Shares. The Journal of Business, v.34, p.411-433, out 1961.

MONTGOMERY, Douglas C.; RUNGER George C. Estatística Aplicada e Probabilidade para Engenheiros. $2^{\circ}$ ed. Rio de Janeiro: LTC, 2008.

MORETTIN, Pedro A.; TOLOI, Clélia M. C. Análise de Séries Temporais. São Paulo: Edgard Blucher, 2004.

NETO, Jorge Augusto; SAITO, Richard. Dividend yield e Persistência de Retornos Anormais das Ações: Evidência do Mercado Brasileiro. XXVI ENANPAD, Anais. Salvador 2002.

SAMUELSON, P. A. Proof that properlyanticipated prices fluctuate randomly. Industrial Management Review, Cambridge: Massachusetts Institute Technology, v. 6, p. 41- 49, Spr. 1965.

SCHLITZER, G. Testing de null of stationarity against the alternative of unit root: an application to the Italian post-war. Applied Economics, V.28, pg. 327-331, 1996.

SHEFRIN, Hersh M.; STATMAN, Meir. Explaining investor, Preference for Cash Dividends. Journal of Finance Economics. Amsterdam: North-Holland. P.252-282, v13, n.2, jun.1984.

SPERANZINI, Milton Medeiros. Efeitos da Política de Dividendos Sobre o Valor das ações no Mercado Brasileiro de Capitais. São Paulo. Dissertação (Mestrado) Faculdade de Economia, Administração e Contabilidade da Universidade de São Paulo - DEA/USP, 1994.

WALTER, James E. Dividend Policy: its influence on the value of the enterprise. The Journal of Finance. New York: The American Finance Association, v.18, n.2, p.280291, mai. 1963. 